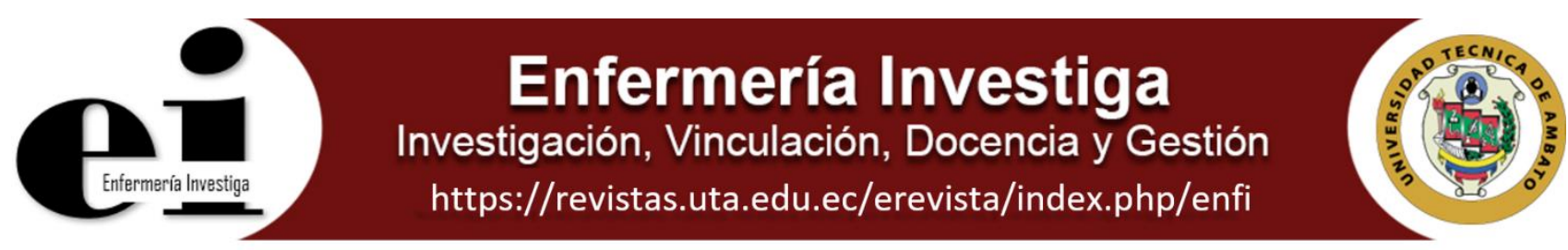

\title{
COMPLICACIONES EN PACIENTES DIABÉTICOS CON COVID-19
}

\section{COOMPLICATIONS IN DIABETIC PATIENTS WITH COVID-19}

Barrionuevo Mejía Jenny ${ }^{1}$ https://orcid.org/0000-0001-7667-1538, López Pérez Grace ${ }^{1}$ https://orcid.org/0000-0002-0558-5429

'Universidad Técnica de Ambato, Facultad de Ciencias de la Salud, Carrera de Enfermería

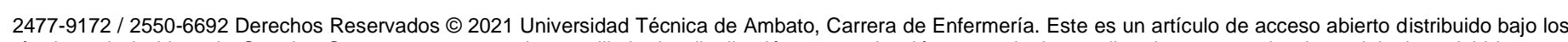

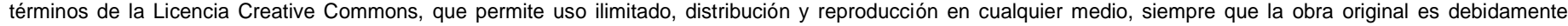
citada.

\section{RESUMEN}

Introducción: Durante la pandemia por COVID-19 la población diabética ha sido considerada como vulnerable y de alto riesgo al ser más propensos a adquirir infecciones respiratorias por deterioro del sistema inmunológico y alteración de los niveles de glucosa. Objetivo: Describir las complicaciones en pacientes diabéticos con COVID-19. Métodos: revisión sistemática de artículos publicados en el periodo 2015-2020 en bases de datos como: Medline, PubMed, Lilacs, Scielo, Redalyc, Elsiever, BVS y Google académico mediante descriptores como: Diabetes, COVID-19, coronavirus, infecciones del sistema respiratorio, además del uso de combinaciones entre dichos descriptores. Etapas de la revisión sistemática: la primera de búsqueda de documentos, la segunda de selección, la tercera de extracción de información y la cuarta presentación de resultados. Resultados: Los mecanismos potenciales que pueden aumentar la susceptibilidad a COVID-19 en pacientes diabéticos: incluyen una mayor unión celular de SARS-CoV-2 a la enzima convertidora de angiotensina-2 (ACE2) en células alveolares, miocardio, riñón y páncreas, una disminución del aclaramiento viral, una disminución en la función de las células $\mathrm{T}$, un aumento en la hiperinflamación y el síndrome de tormenta de citoquinas. Conclusiones: Las principales complicaciones de los pacientes diabéticos con COVID-19 son: síndromes de distrés respiratorio severos, síndrome de hiperinflamación sistémica extrapulmonar, shock, vasoplejia, insuficiencia respiratoria, colapso cardiopulmonar, neumonía grave, miocarditis, lesión renal aguda, daño pancreático, esto debido a mecanismos inmunológicos y metabólicos que conllevan a un mayor riesgo de muerte

Palabras Claves: diabetes, COVID-19, coronavirus, infecciones del sistema respiratorio

\begin{abstract}
Introduction: During the COVID-19 pandemic, the diabetic population has been considered vulnerable and at high risk as they are more prone to acquiring respiratory infections due to deterioration of the immune system and alteration of glucose levels. Objective: To describe the complications in diabetic patients with COVID-19. Methods: systematic review of articles published in the 2015-2020 period in databases such as: Medline, PubMed, Lilacs, Scielo, Redalyc, Elsiever, BVS and academic Google using descriptors such as: Diabetes, COVID19 , coronavirus, system infections respiratory, in addition to the use of combinations between these descriptors. Stages of the systematic review: the first to search for documents, the second to select, the third to extract information and the fourth to present the results. Results: Potential mechanisms that may increase susceptibility to COVID-19 in diabetic patients: include increased cellular binding of SARS-CoV-2 to angiotensinconverting enzyme-2 (ACE2) in alveolar cells, myocardium, kidney and pancreas, a decrease in viral clearance, a decrease in T-cell function, an increase in hyperinflammation, and cytokine storm syndrome. Conclusions: The main complications of diabetic patients with COVID-19 are: severe respiratory distress syndromes, extrapulmonary systemic hyperinflammation syndrome, shock, vasoplegia, respiratory failure, cardiopulmonary collapse, severe pneumonia, myocarditis, acute kidney injury, pancreatic damage, this due to immunological and metabolic mechanisms that lead to an increased risk of death
\end{abstract}

Keywords: diabetes, COVID-19, coronavirus, respiratory system infections 


\section{INTRODUCCIÓN}

La enfermedad por coronavirus 2019 (COVID-19) ha provocado una alta tasa de morbi-mortalidad a nivel mundial, especialmente en grupos vulnerables como los pacientes diabéticos, donde existe un 2,61 mayor probabilidad de desarrollar enfermedad grave al infectarse con el virus del síndrome respiratorio agudo severo tipo-2 (SARS-CoV-2) y 2,5 veces el riesgo de mortalidad asociada a COVID-19. De acuerdo a estudios preliminares desarrollados en países asiáticos, alrededor del $10 \%$ de los pacientes con diabetes y COVID-19 sufrieron al menos un episodio de hipoglucemia (glicemia $<70 \mathrm{mg} / \mathrm{dl}$ ), en el curso de la enfermedad, con una mayor susceptibilidad a enfermedades infecciosas y mortalidad por alteraciones de la respuesta inmune, relacionada con la hiperglucemia particularmente en adultos mayores, con diabetes mellitus tipo 2 (DM2), obesidad e hipertensión arterial (HTA) (1-2)

En China, Guan et al., en un estudio que incluyó a 1099 pacientes con COVID-19, encontró que 173 tenían enfermedad grave, de los cuales el $16,2 \%$ tenía DM2, seguido por enfermedades coronarias $(5,8 \%)$, HTA $(3,7 \%)$ y enfermedad cerebrovascular $(2,3 \%)(3)$

Las complicaciones en pacientes diabéticos con COVID-19 es de gran relevancia, ya que permitirá identificar cómo la diabetes conlleva a una mayor vulnerabilidad a enfermarse gravemente al infectarse por SARS-CoV-2, pues como lo expone Hernández (4), al desarrollar COVID-19, es más difícil tratar las variaciones en los niveles de glucosa y las alteraciones propias de la diabetes, lo que complica la evolución intrahospitalaria y conduce a una recuperación más lenta.

La inflamación crónica, el aumento de la actividad de coagulación, el deterioro de la respuesta inmune y el posible daño pancreático directo por SARS-CoV-2 estarían entre los mecanismos subyacentes relacionados a la evolución desfavorable de COVID-19 en DM2. Los mecanismos potenciales que pueden aumentar la susceptibilidad a COVID19 incluyen una mayor unión celular de SARS-CoV-2 a la enzima convertidora de angiotensina-2 (ACE2) en células alveolares, miocardio, riñón y páncreas, una disminución del aclaramiento viral, una disminución en la función de las células $\mathrm{T}$, un aumento en la hiperinflamación y el síndrome de tormenta de citoquinas (5)

En este sentido, el presente estudio tiene como objetivo describir las complicaciones en pacientes diabéticos con COVID-19, con lo que se pueda tener una línea base que permita que el personal de salud reconocer la vulnerabilidad de esta población y se pueda llevar a cabo planes de acción en favor de este colectivo.

\section{MÉTODOS}

Se trata de un meta-análisis con artículos, publicaciones, revisiones y documentos de sociedades científicas para conocer ¿Cuáles son las complicaciones del COVID-19 en pacientes diabéticos?

Búsqueda de datos: se realizó a través de Google scholar y bases de datos como PUBMED, Lilacs, Scielo, Elsiever, Latindex, BVS y entre otras fuentes mediante descriptores como: Diabetes, COVID-19, coronavirus, SARS-CoV-2, infección respiratoria, además del uso de combinaciones entre dichos descriptores. La recolección de información se realizó en el periodo $2015-2020$.

Selección: como criterios de inclusión se consideraron: revisiones sistemáticas, artículos originales e informes de instituciones de salud relacionados con los descriptores (diabetes, COVID-19, coronavirus, Infecciones del Sistema Respiratorio). Fueron excluidas: publicaciones con deficiencias metodológicas de acuerdo a criterios PRISMA (Preferred Reporting Items for Systematic reviews and MetaAnalyses, QUOROM (Quality of Reporting of Meta-analysis), ECA (ensayos clínicos aleatorizados), RS (revisiones sistemáticas), información que no correspondía a los últimos 5 años y que se encuentren en idiomas diferentes al español e inglés (5).

El diagrama de selección se muestra a continuación:

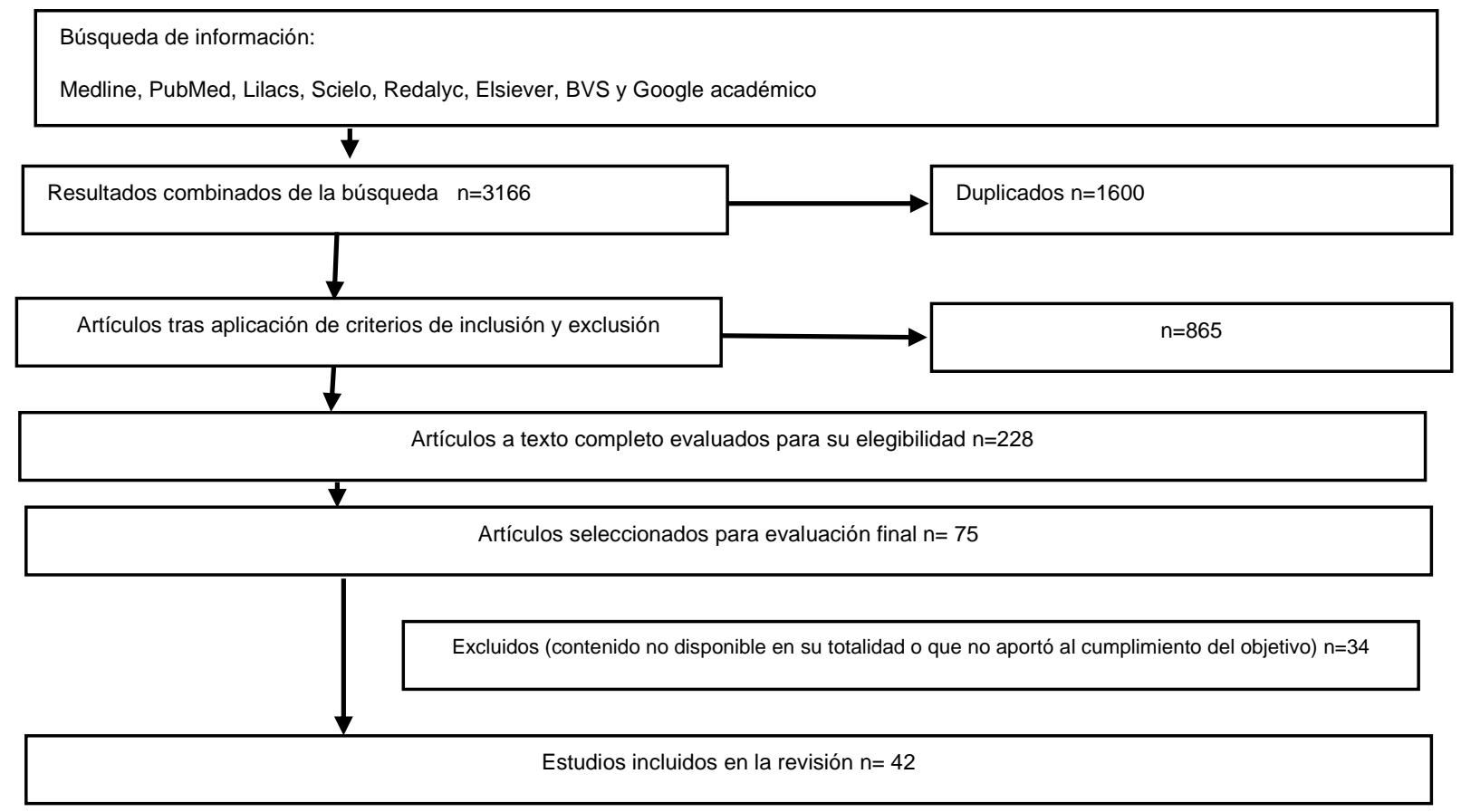


La extracción de datos: una vez seleccionados los artículos se procedió a extraer datos como: título, autor, año, metodología y resultados para determinar la consistencia metodológica y el aporte al cumplimiento de objetivos.

Presentación de resultados: Los resultados de las investigaciones seleccionadas se presentaron en un cuadro que contiene título, autor, año, metodología y resultados que contribuyeron a dar respuesta a la interrogante planteada en la presente revisión sistemática.

\section{RESULTADOS}

Para el desarrollo de revisión sistemática: se contó con un total de 42 artículos mismos que permitieron dar cumplimiento a los objetivos planteados, estos se detallan a continuación:

Tabla 1. Artículos científicos seleccionados para la revisión

\begin{tabular}{rll}
\hline Título & & Autor \\
\hline 1. & Diabetes in COVID- \\
& Awadhesh, \\
& $\begin{array}{l}\text { pathophysiology, } \\
\text { prognosis } \\
\text { practical } \\
\text { considerations. }\end{array}$ & Amesh, \\
& Anoop, M., $(7)$. \\
&
\end{tabular}

\section{Año Metodología Resultados}

K., 2020 Revisión

G.,

\&
considerations.
Existe evidencia de una gravedad de COVID-19 en pacientes con diabetes. COVID-19 podría tener efecto sobre la fisiopatología de la diabetes. El control de la glucosa en sangre es importante no solo para los pacientes infectados con COVID-19, sino también para aquellos que no tienen la enfermedad. $\begin{array}{llll}\text { 2. Diabetes and COVID- } & \text { Kosinski, } & \text { C., } 2020 & \text { Descriptivo } \\ & \text { Zanchi, } & \text { A., } & \end{array}$ Wojtusciszyn, A (8)

3. COVID-19, diabetes Rimesh, mellitus and ACE2: Bhansali, A (9) The conundrum

4. Diabetes mellitus e CoV-2: riesgo, curso e indicaciones de actuación

La diabetes es un factor de riesgo de infección por SARS-CoV-2, asociada con un curso más grave

Los pacientes con diabetes mellitus tienen un mayor riesgo de enfermedad grave y fatal de COVID-19 Entre los pacientes hospitalizados a causa de la COVID-19, un 6-15\% tienen diabetes mellitus y en el grupo de pacientes que requieren tratamiento en la unidad de cuidados intensivos (UCl) o cuya infección conduce a la muerte, este porcentaje es del $20-33 \%$

5. Clinical
characteristics and
outcomes of patients
with diabetes and
COVID-19 in
Association with
glucose-Lowering
medication

Chen, Y., Yang, 2020 Retrospectiva

Los pacientes mayores con diabetes son más propensos a muerte relacionada con COVID19.

D., Cheng, B.,

Chen, J., Peng,

A., Yang, C., Liu,

C., Xiong, M.,

Deng, A (11)

Fernández

Puentes, A., \& obesidad, diabetes e Puentes, A.,
ingreso en UCI en García, M.(12) pacientes COVID-19

$\begin{array}{llllll}\text { 7. Diabetes is a risk Guo, W., Li, M., } & 2020 \\ 48\end{array}$ 


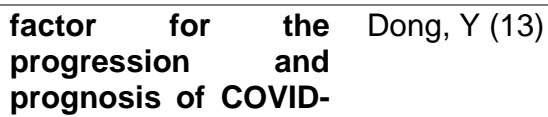

19 y diabetes tienen mayor riesgo de neumonía grave, tormenta citocínica y estado hipercoagulabilidad asociado con la desregulación del metabolismo de la glucosa. from COVID-19
8. Diabetes and
Hartmann, Morris, Goyder, Kinton, J., Perring, J., Nunan, D., \& Khunti, K (14)
J., 2020 Descriptivo

E.,

C., metabolismo de la glucosa. personas con diabetes tienen un mayor riesgo de contraer enfermedades graves por COVID-19, porque se ha evidenciado que el estado proinflamatorio propio de la diabetes, con un aumento en los niveles de IL-6 y proteína $C$ reactiva, puede favorecer la tormenta de citocinas y la respuesta inflamatoria sistémica, que acompaña al síndrome de Distrés respiratorio agudo en pacientes con COVID19

\section{Por qué los Hernández A. (4) 2019 Descriptivo diabéticos son grupo de riesgo frente a la infección COVID-19.}

La expresión de la enzima convertidora de angiotensina-2 en el páncreas (principalmente en células de los islotes) es mayor que en los pulmones, por lo que el SARS-CoV-2 puede unirse a este receptor e ingresar a las células $\beta$ del páncreas produciendo disfunción celular con hiperglucemia aguda elevada en sangre

La diabetes es un predictor significativo de la morbimortalidad en pacientes con COVID-19. La inflamación crónica, el aumento de la actividad de la coagulación, el deterioro de la respuesta inmune y el posible daño pancreático directo por el SARS-CoV-2, se encuentran entre los mecanismos subyacentes de la asociación entre la diabetes y el COVID-19

Los pacientes con diabetes tienen un mayor riesgo de complicaciones y de muerte relacionada con COVID-19. La edad avanzada, la multimorbilidad, la hiperglucemia, la lesión cardíaca y la respuesta inflamatoria grave son 


\begin{tabular}{|c|c|c|c|c|}
\hline & & & & $\begin{array}{l}\text { predictores de un mal } \\
\text { pronóstico. }\end{array}$ \\
\hline $\begin{array}{l}\text { 12. Is diabetes } \\
\text { associated } \\
\text { mortality with } \\
\text { severity of COVID- } \\
\text { 19? }\end{array}$ & $\begin{array}{l}\text { Kumar, A., Arora, } \\
\text { A., Sharma, P., } \\
\text { Anikhindi, S., } \\
\text { Bansal, N., Singla, } \\
\text { V, Srivastava, } \\
\text { A.(16) }\end{array}$ & 2020 & Revisión & $\begin{array}{l}\text { La diabetes en pacientes } \\
\text { con COVID-19, se asocia } \\
\text { con un aumento de la } \\
\text { gravedad y mortalidad y de } \\
\text { COVID-19, } \\
\text { comparación con los no } \\
\text { diabéticos. }\end{array}$ \\
\hline $\begin{array}{l}\text { 13. Diabetes in COVID- } \\
\text { 19: Prevalence, } \\
\text { pathophysiology, } \\
\text { prognosis and } \\
\text { practical } \\
\text { considerations }\end{array}$ & $\begin{array}{l}\text { Kumar, A., Gupta, } \\
\text { R., Ghosh, A., \& } \\
\text { Misra, A (17) }\end{array}$ & 2020 & Revisión & $\begin{array}{l}\text { Existe una mayor } \\
\text { incidencia y gravedad de } \\
\text { COVID-19 en pacientes } \\
\text { con diabetes, debido al } \\
\text { aumento de la expresión } \\
\text { de ACE-2 e incremento de } \\
\text { una proteasa tipo furina, } \\
\text { que facilita la replicación } \\
\text { viral }\end{array}$ \\
\hline $\begin{array}{l}\text { 14. COVID-19 } \\
\text { diabetes }\end{array}$ & $\begin{array}{l}\text { Ma, R., Holt, } R \\
(18)\end{array}$ & 2020 & Descriptivo & $\begin{array}{l}\text { Los pacientes con diabetes } \\
\text { tienen una mayor } \\
\text { susceptibilidad a COVID- } \\
19 \text {, con mayor riesgo de } \\
\text { enfermedad grave }\end{array}$ \\
\hline $\begin{array}{l}\text { 15. Dabetes and COVID- } \\
19\end{array}$ & Zachary, T (19) & 2020 & Descriptivo & $\begin{array}{l}\text { La diabetes incrementa la } \\
\text { gravedad de la COVID-19 } \\
\text { con mayor inflamación } \\
\text { pulmonar e infiltrados. }\end{array}$ \\
\hline $\begin{array}{l}\text { 16. Crisis hiperglicémica } \\
\text { aguda como debut } \\
\text { de diabetes mellitus } \\
\text { y COVID-19 ¿Una } \\
\text { nueva causa de } \\
\text { diabetes? }\end{array}$ & $\begin{array}{l}\text { Gómez a, Del } \\
\text { Castillo S, Chiu, E, } \\
\text { Salsavilca E (20) }\end{array}$ & 2020 & Análisis de casos & $\begin{array}{l}\text { La infección por COVID-19 } \\
\text { puede precipitar } \\
\text { aparición da de crisis } \\
\text { hiperglicémica } \\
\text { pacientes diabéticos. }\end{array}$ \\
\hline $\begin{array}{l}\text { 17. COVID-19 and } \\
\text { diabetes }\end{array}$ & $\begin{array}{l}\text { Paquot, } \quad \text { N., } \\
\text { Randermecker, R } \\
(21)\end{array}$ & 2020 & Descriptivo & $\begin{array}{l}\text { El ingreso de pacientes } \\
\text { diabéticos a la unidad de } \\
\text { cuidados intensivos por } \\
\text { COVID-19, es dos a tres } \\
\text { veces mayor que la } \\
\text { observada en pacientes no } \\
\text { diabéticos y el riesgo de } \\
\text { progresar a enfermedad } \\
\text { crítica o fatal, se } \\
\text { incrementa en con una } \\
\text { probabilidad de } 3 \text { a } 4 \\
\text { veces mayor en los } \\
\text { pacientes con diabetes. }\end{array}$ \\
\hline $\begin{array}{l}\text { 18. COVID-19 and } \\
\text { diabetes: a collision } \\
\text { and collusion of two } \\
\text { diseases }\end{array}$ & $\begin{array}{lr}\text { Feldman, } & \text { E., } \\
\text { Savelieff, } & \text { M., } \\
\text { Hayek, } & \text { S., } \\
\text { Pennathur, } & \text { S., } \\
\text { Kretzler, M., } & \text { Pop, } \\
\text { R (22) } & \end{array}$ & 2020 & Descriptivo & $\begin{array}{l}\text { Los pacientes con diabetes } \\
\text { son susceptibles a } \\
\text { complicaciones inducidas } \\
\text { por la COVID-19 }\end{array}$ \\
\hline $\begin{array}{l}\text { 19. COVID-19 } \\
\text { diabetes: } \\
\text { bidirectional } \\
\text { relationship }\end{array}$ & $\begin{array}{l}\text { Lima, M., Carrera, } \\
\text { C., Madera, M., } \\
\text { Marín, } \\
\text { Contreras, M (23) }\end{array}$ & 2020 & Descriptivo & $\begin{array}{l}\text { Los diabéticos infectados } \\
\text { con SARS-CoV-2 tienen } \\
\text { una tasa más alta de } \\
\text { ingreso hospitalario, } \\
\text { neumonía grave y una } \\
\text { mayor mortalidad en } \\
\text { comparación con los } \\
\text { sujetos no diabéticos. }\end{array}$ \\
\hline $\begin{array}{l}\text { 20. Diabetes is } \\
\text { associated with } \\
\text { increased risk for in- } \\
\text { hospital mortality in }\end{array}$ & $\begin{array}{l}\text { Palaiodimos, L., } \\
\text { Chamorro, } \\
\text { Karamanis, D., Li, } \\
\text { W., Zavras, P., }\end{array}$ & 2020 & Revisión & $\begin{array}{l}\text { Este metaanálisis muestra } \\
\text { que la probabilidad de } \\
\text { muerte es un } 65 \% \text { mayor } \\
\text { en pacientes diabéticos }\end{array}$ \\
\hline
\end{tabular}


patients with COVID19: a systematic review and metaanalysis comprising 18,506 patients.

21. Diabetes mellitus in Medina the context of Hernández E (25) COVID-19
Priyanka, M., \& Kokkimidis,

(24)

D. hospitalizados con COVID19.

2020 Revisión

2020 Revisión
22. COVID-19 pandemic, Ranganath, M., \& 2020 Transversal coronaviruses, and Sriram, G (26) diabetes mellitus
La desregulación del
sistema inmunitario y el
estado de inflamación
persistente en pacientes
con diabetes mellitus tipo 2
y COVID-19, provocan un
mayor riesgo de
desarrollar complicaciones,
en comparación con las
personas no diabéticas

La edad avanzada y la presencia de diabetes mellitus, hipertensión arterial y obesidad aumentan

significativamente el riesgo de hospitalización y muerte en pacientes con COVID19
23. Diabetes and COVID- Jeong, I., Yoon, 2020 Descriptivo 19: global and K., Lee, M (27) regional perspective

Los pacientes con diabetes son vulnerables a las infecciones debido a la hiperglucemia; función inmunológica deteriorada; complicaciones

vasculares; $\quad y$ comorbilidades tales como hipertensión, dislipidemia y enfermedad cardiovascular.
24. COVID-19 and Rimesh, P., \& 2020 Revisión diabetes mellitus: An Sanjay, K (28) unholy interaction of two pandemics.
La diabetes se caracteriza por un estado proinflamatorio, que contribuye a la replicación del SARS-CoV-2 y a una respuesta proinflamatoria prolongada, que predispone a una forma de la enfermedad severa y letal.

25. Manejo de pacientes Salazar J. (29) 2020 Descriptivo
con diabetes en el
contexto de la
Pandemia CoVID-19.

La diabetes mellitus tipo 2 (DM2), ha sido identificada como un factor de riesgo para desarrollar un cuadro más grave de COVID-19; siendo mayor el riesgo si es un paciente de reciente diagnóstico

en comparación a alguien bajo manejo previo

La microangiopatía en la diabetes mellitus altera la distensibilidad pulmonar con la afectación del intercambio gaseoso, predisponiendo a complicaciones graves en la COVID-19 
27. Diabetes and COVID19 : a major challenge in pandemic period
Chakraborty, C

Sharma, A.,

Bhattacharya, M.,

Sharma, G.,

Agoramoorthy, G.,

Lee, $s$ (31)

28. COVID-19 and

diabetes mellitus: the

link and clinical implications
La diabetes es una comorbilidad grave en pacientes con COVID-19

$\begin{array}{lll} & 2019 \text { Revisión } & \text { La diabetes es una } \\ \text { comorbilidad grave en } \\ \text { pacientes con COVID-19 }\end{array}$

2020 Descriptivo

,

Descriptivo La diabetes mellitus es una ,

(20)

La diabetes mellitus es una
de las enfermedades no

transmisibles más comunes asociadas con el empeoramiento del estado

$\begin{array}{ll}\text { Ugwueze } & \mathrm{C}, \\ \text { Ezeokpo } & \mathrm{B},\end{array}$

Nnolim b, Agim E,

Anilkpo C,

Onyekachi E (32)
29. Diabetes and COVID19: a global health challenge
Shenoy, A., 2020 Revisión Ismaily, M., Bajaj, $\mathrm{M}$ (33) clínico en pacientes con COVID-19

Los pacientes con diabetes y COVID-19 severo, tienen un incremento de las citocinas proinflamatorias y una tasa de supervivencia peor que los no diabéticos

30. COVID-19 and Desai, R., Singh, 2020 Revisión diabetes mellitus: a S., Parekh, T., need for prudence in Sachdeva, S., elderly patients from Sachdeva, R., a pooled analysis Kumar, G (34)

31. Covid-19 and Lim S, Hyuk j, 2020 Retrospectiva diabetes mellitus: Sang $H$, Nauck $M$ from pathophysiology to clinical management (35)

La prevalencia global de diabetes en pacientes con COVID-19 se encontró que era del $13,2 \%$

La hiperglucemia 0 antecedentes de diabetes son predictores de morbilidad y mortalidad en pacientes con

SARS-CoV-2

32. Consecuencias de la COVID-19 sobre las personas con diabetes

Bellido, V; Pérez, 2020 Descriptiva A (36)

El estado de inflamación crónica, el deterioro de respuesta inmune y alteración de la coagulación, son los mecanismos fisiopatológicos que contribuyen al aumento de la morbimortalidad de la COVID-19 en personas diabéticas.

33. Diabetic patients with COVID-19 infection are at higher risk of $\mathrm{UCl}$ admission and poor short term outcome

Roncón, L; Zuin, 2020 Revisión M; Rigatelli, G; sistemática Zuliani, G (37)

Los pacientes diabéticos con COVID-19 tienen un mayor riesgo de ingreso en la $\mathrm{UCl}$ e incremento de la mortalidad.
34. Manejo de la diabetes mellitus en tiempos de COVID-19

Los reportes disponibles describen que los pacientes con diabetes mellitus presentan un mayor riesgo de evolución desfavorable y desarrollo de complicaciones e incluso aumento de la tasa de mortalidad.

$\begin{array}{lrlrl}\text { 35. Infección } & \text { por } & \text { Torres, } \quad M ; 2020 & \text { Descriptiva } \\ \text { coronavirus } & \text { en } & \text { Caracas, N; Peña, } \\ \text { pacientes } & \text { con } & \text { B; Juárez, J; } \\ \text { diabetes } & & \text { Medina, } \\ & \text { Martínez, M (39) }\end{array}$

Se ha documentado una progresión más severa y riesgo más alto de mortalidad de 2.3 veces, en pacientes diabéticas con COVID-19. 


\begin{tabular}{|c|c|c|c|c|c|}
\hline & $\begin{array}{l}\text { COVID-19 and } \\
\text { diabetes: a complex } \\
\text { bidirectional } \\
\text { relationship }\end{array}$ & $\begin{array}{lr}\text { Muniangi, } & \text { H., } \\
\text { Akalestou, } & \text { E., } \\
\text { Salem, V., } & \text { Misra, } \\
\text { S., Oliver, } & \text { N., } \\
\text { Rutter, G (40) }\end{array}$ & 2020 & Descriptiva & $\begin{array}{l}\text { La infección por SARS- } \\
\text { CoV-2 exacerba la la } \\
\text { hiperglucemia en personas } \\
\text { con diabetes. }\end{array}$ \\
\hline 37. & $\begin{array}{l}\text { COVID-19: is there a } \\
\text { link between the } \\
\text { course of infections } \\
\text { and pharmacological } \\
\text { agents in diabetes? }\end{array}$ & $\begin{array}{ll}\text { Filardi, } & \text { T., } \\
\text { Morano, S (41) }\end{array}$ & 2020 & Revisión & $\begin{array}{l}\text { El COVID-19 puede tener } \\
\text { un desenlace fatal en } \\
\text { personas con diabetes, al } \\
\text { desarrollar neumonía } \\
\text { grave especialmente en } \\
\text { adultos mayores }\end{array}$ \\
\hline 38. & $\begin{array}{l}\text { Diabetes, infection } \\
\text { risk and COVID-19 }\end{array}$ & Erener, S (42) & 2020 & Revisión & $\begin{array}{l}\text { Los } \\
\text { epidemiológicos muestran } \\
\text { que la diabetes mal } \\
\text { controlada es un factor de } \\
\text { riesgo, para diversas } \\
\text { enfermedades infecciosas } \\
\text { como el COVID-19. }\end{array}$ \\
\hline \multirow[t]{2}{*}{39.} & $\begin{array}{l}\text { Diabetes and COVID- } \\
19\end{array}$ & $\begin{array}{l}\text { Bouhanick; } \\
\text { Cracowski, } \\
\text { Faillie, J (43) }\end{array}$ & 2020 & Descriptivo & $\begin{array}{l}\text { Los pacientes diabéticos } \\
\text { con COVID-19 tienen un } \\
\text { alto riesgo de neumonía } \\
\text { grave y presentan un } \\
\text { marcado rado }\end{array}$ \\
\hline & & & & & $\begin{array}{ll}\text { proinflamatorio } & \text { y } \\
\text { protrombótico } & \text { en } \\
\text { comparación } & \text { con los } \\
\text { pacientes no } & \text { diabéticos } \\
\text { infectados. } & \end{array}$ \\
\hline 40. & $\begin{array}{l}\text { Diabetes and COVID- } \\
\text { 19: disease- } \\
\text { management-people }\end{array}$ & $\begin{array}{l}\text { Peric, S; Stulning, } \\
\text { T (44) }\end{array}$ & 2020 & Descriptivo & $\begin{array}{l}\text { La diabetes predispone a } \\
\text { un curso severo de la } \\
\text { COVID-19 y duplica el } \\
\text { riesgo de mortalidad, } \\
\text { debido a afectación } \\
\text { pulmonar, cardiaca y } \\
\text { comorbilidades }\end{array}$ \\
\hline 41. & $\begin{array}{l}\text { Diabetes and COVID- } \\
19: \text { a systematic } \\
\text { review on the current } \\
\text { evidences }\end{array}$ & $\begin{array}{l}\text { Abdi, A; Jalilian, } \\
\text { M; Ahmadi, P; } \\
\text { Vlaisavljevic, } \quad \text { Z } \\
\text { (45) }\end{array}$ & 2020 & Revisión & $\begin{array}{l}\text { La diabetes es un factor de } \\
\text { riesgo y contribuye a la } \\
\text { gravedad y mortalidad de } \\
\text { los pacientes con COVID- } \\
19\end{array}$ \\
\hline 42. & $\begin{array}{l}\text { COVID-19 } \\
\text { diabetes }\end{array}$ & $\begin{array}{l}\text { Villabona, Carmen } \\
\text { V. (46) }\end{array}$ & 2020 & Reflexión & $\begin{array}{l}\text { La hipoglucemia es un } \\
\text { factor de pronóstico } \\
\text { negativo para pacientes } \\
\text { diabéticos con COVID-19. }\end{array}$ \\
\hline
\end{tabular}

\section{DISCUSIÓN}

Diversas investigaciones han abordado el tema del COVID-19 y la diabetes debido a su repercusión sobre la salud pública, porque se ha establecido que la población con mayor edad o que tiene asociación a patologías crónicas como la diabetes, es propensa a desarrollar formas clínicamente más graves (5) , pues cualquiera que sea la etiología subyacente, las personas con diabetes tienen un mayor riesgo de enfermedad de COVID19 grave y fatal (6) al desarrollar infecciones complejas de difícil manejo (7)

Se confirma este criterio con la evidencia de una mayor necesidad de ingreso a Unidades de Cuidados Intensivos por COVID-19 en pacientes con diabetes. De acuerdo a varias investigaciones, la población diabética mayor a los 65 años con COVID-19 se ha considerado con mayor mortalidad, así por ejemplo en China se reportaron 72,314 casos, es decir que hubo una incidencia aumentada de mortalidad en este tipo de pacientes del $2,3 \%$ al $7,3 \%$ (8).

Hartmann et al., en una investigación de 1.590 pacientes hospitalizados en China en 575 hospitales la diabetes aumentó significativamente el riesgo de complicaciones y muerte en infección por COVID-19, debido a varios mecanismos como: niveles elevados de citocinas pro-inflamatorias, desequilibrio de la enzima convertidora de angiotensina 2, depuración viral reducida e incremento de la resistencia a la insulina (14).

El estudio desarrollado en la India por Katulanda, et al., (2) donde se concluye que los pacientes con COVID-19 y diabetes tienen más probabilidades de desarrollar síndromes respiratorios, ingreso a $\mathrm{UCl}$ por requerir ventilación invasiva, donde la diabetes es un determinante de la gravedad y la mortalidad de los pacientes con COVID-19. 
De igual forma Bellido, et al., (36) establece que la diabetes es un factor de riesgo de mal pronóstico para el COVID-19, ya que existe un estado de inflamación crónica, deterioro de la respuesta inmune y alteración de la coagulación, mecanismos que contribuyen a un incremento en la morbimortalidad ante el COVID-19. Además, se ha descrito un posible daño directo sobre el páncreas por el SARS-CoV-2, que empeora aún más la hiperglucemia, razón por la cual la probabilidad de desarrollar un cuadro severo. La diabetes predispone a un curso particularmente severo de la enfermedad y duplica el riesgo de mortalidad por COVID-19 debido a la afectación pulmonar y cardíaca (11-16). Además, la disminución del aclaramiento viral y la reducción en la función de las células $T$, aumentan la susceptibilidad a COVID-19 en personas con diabetes, así también la expresión aumentada del receptor tipo 2 de la enzima convertidora de angiotensina en las células alveolares, miocardio, riñón y páncreas favorecen una mayor unión celular del SARS-CoV-2 en este tipo de pacientes $(17,18)$. Rimesh et al., (9) establecen que la inmunidad innata comprometida, el incremento de las citocinas proinflamatorias y la expresión reducida de ACE2, contribuyen a un pronóstico desfavorable en COVID-19

Los diabéticos infectados con SARS-CoV-2 tienen una tasa más alta de ingreso hospitalario, neumonía grave y una mayor mortalidad en comparación con los sujetos no diabéticos (20) porque la diabetes empeora la gravedad de la enfermedad, con mayor inflamación pulmonar e insuficiencia respiratoria (21). Por lo tanto, es imperativo que las personas con diabetes tengan un peor pronóstico porque la hiperglucemia 0 antecedentes de la misma constituyen predictores de morbilidad y mortalidad en pacientes con COVID-19, por complicaciones respiratorias (patología pulmonar grave), causadas por la rápida proliferación viral que conduce a síndromes respiratorios agudos y severos, fibrosis pulmonar, daño pulmonar agudo, además de complicaciones cardiovasculares como: tromboembolismo, coagulación intravascular diseminada, daño endotelial y eventos cardiovasculares por incrementos en la viscosidad sanguínea (22)

Gómez et al., (20) y Medina, et al. (25), plantean que la desregulación del metabolismo de la glucosa facilita la entrada y proliferación del SARS-CoV-2 y ocasiona complicaciones como neumonía severa, lesión pulmonar y síndrome de enfermedad respiratoria aguda, que conllevan al ingreso a UCI por necesidad de ventilación mecánica y evolución a la mortalidad (24).

Los pacientes con diabetes son vulnerables a las infecciones por coronavirus debido a la hiperglucemia, función inmunológica deteriorada, complicaciones vasculares y comorbilidades tales como hipertensión, dislipidemia y enfermedad cardiovascular, condiciones que exacerban la gravedad del COVID-19, por esto se reconoce a la diabetes como una de las comorbilidades más graves de la COVID-19 (25-30). Los pacientes diabéticos infectados con COVID-19 presentan mayores complicaciones cardiovasculares como: la lesión en el miocardio, disfunción cardiaca, shock por efecto ventilatorio restrictivo, coagulación intravascular diseminada, insuficiencia multiorgánica y muerte. $(25,26)$.

Las condiciones clínicas antes expuestas se explican que los pacientes con diabetes tienen una mayor respuesta inflamatoria ante el COVID-19 y desarrollan complicaciones debido a la presencia de factores de riesgo como la edad avanzada, sobrepeso, presencia de comorbilidades como la hipertensión, enfermedades cardiovasculares, oculares y renales, además de una respuesta inmune deprimida, lo que conlleva a una mayor neutrofilia y linfopenia, incidencia de coagulopatía (niveles más altos de dímero D), neumonía severa) y mayor tasa de mortalidad por empeoramiento de la función cardíaca $(31,32)$.

Desde esta perspectiva para Awadhesh, et al., (7) la diabetes se asocia con consecuencias más fatales ante el contagio de COVID-19, por mecanismos de quimiotaxis de neutrófilos deteriorada, aumento de la expresión de ACE-2, incremento de la furina que facilita la replicación viral, deterioro de la función de las células T y un aumento de las citocinas proinflamatorias, lo que conlleva a un desenlace fatal debido a la gravedad de la enfermedad, razón por la cual el control de la glucosa debe ser la primera línea de atención en pacientes diabéticos infectados por COVID-19 (34).

Por lo tanto, podría indicarse que, las personas con afecciones médicas preexistentes como la diabetes son más vulnerables a enfermarse gravemente al infectarse por COVID-19, porque al desarrollar una infección viral, es más difícil de tratar por las variaciones en los niveles de glucosa en la sangre y complicaciones propias de la patología, ya que la alteración de la glucosa es un factor pronóstico negativo de enfermedad crítica en pacientes con COVID-19 $(35,36)$.

De ahí que se puede establecer que la diabetes y otras comorbilidades son predictores significativos de morbilidad y mortalidad en pacientes con COVID-19, porque la glucemia no controlada es indicativa de gravedad y muerte, porque desencadena condiciones de mayor estrés, con liberación de hormonas hiperglucémicas, como los glucocorticoides y catecolaminas, lo que lleva a un aumento de los niveles de glucosa que complica el cuadro infeccioso (37).

En contraposición a lo expuesto por los estudios presentados, para Ranganath, et al., (26) la diabetes no contribuye de forma independiente un factor de riesgo para el COVID-19, pero los niveles de glucosa en plasma sí son predictores independientes de mortalidad y morbilidad en pacientes con COVID-19; por cinco mecanismos: una mayor afinidad de unión celular a la enzima convertidora de angiotensina-2, la disminución del aclaramiento viral, la disminución de la función de las células $T$, aumento de la susceptibilidad a la hiperinflamación, la tormenta de citocinas y la presencia de enfermedad cardiovascular.

La investigación de Czupryniak (10) establece que los pacientes hospitalizados con COVID-19, entre un 6-15\% tienen diabetes, edad avanzada, alteraciones del sistema inmune y la presencia de complicaciones crónicas como las enfermedades cardiovasculares y renales, por lo tanto, la diabetes no parece ser un factor de riesgo por sí solo, sino más bien una comorbilidad muy importante (40). Chen, et al., (11) y Filardi, et al., quienes tras analizar casos de gravedad y mortalidad de COVID-19 en pacientes diabéticos, la edad avanzada es un factor determínate asociado a la diabetes (41)

Finalmente, los factores de riesgo de las complicaciones de la COVID-19, son es la diabetes asociada a la edad avanzada e hipertensión arterial, que se asocian al curso grave de la infección por SARS-CoV-2. 


\section{CONCLUSIÓN}

La diabetes es una de las comorbilidades mayormente asociadas a gravedad y mortalidad ante COVID-19, debido a la presencia de factores de riesgo de otras enfermedades como la hipertensión arterial, patologías cardiovasculares, oculares y renales, además de una respuesta inmune deprimida.

Los mecanismos por los cuales los pacientes diabéticos son propensos a desarrollar estados más graves de COVID-19, son la mayor afinidad de unión celular a la enzima convertidora de angiotensina-2, la disminución del aclaramiento viral, la disminución de la función de las células $\mathrm{T}$, aumento de la susceptibilidad a la hiperinflamación, la tormenta de citocinas y la presencia de enfermedad cardiovascular.

La morbimortalidad por COVID-19 en pacientes diabéticos se relacionan con complicaciones graves como: síndromes de distrés respiratorio severos y síndrome de hiperinflamación sistémica extrapulmonar, shock, vasoplejia, insuficiencia respiratoria, colapso cardiopulmonar, neumonía grave, miocarditis, lesión renal aguda, daño pancreático.

Las complicaciones en personas diabéticas que contraen COVID-19 son severas, tiene que ver en primer lugar con el hecho que el sistema inmunológico esté comprometido, lo que dificulta la lucha contra el virus y probablemente conduce a un período de recuperación más prolongado y, en segundo lugar, porque el virus puede prosperar rápidamente en un entorno de glucosa en sangre elevada. Sin embargo, se debe tomar en cuenta que debido a que el COVID-19 es una enfermedad relativamente nueva aún existen contradicciones y afirmaciones aleatorias en torno a los procesos de morbimortalidad por esta causa.

Por lo que se sugiere:

- Establecer nuevas líneas de investigación sobre la asociación de la diabetes y el COVID-19, así como de otras comorbilidades.

- Difundir los resultados de la presente investigación en el medio sanitario pues al ser un estudio preliminar servirá como línea base para futuras investigaciones e intervenciones en favor de la protección de este grupo humano altamente vulnerable.

\section{CONFLICTOS DE INTERÉS}

Los autores declaran no tener conflictos de interés, ni fuentes de financiamiento

\section{REFERENCIAS}

1. Hussain A, Bhowmik B, do Vale Moreira NC. COVID-19 and diabetes: Knowledge in progress. Diabetes Res Clin Pract. 2020; 162:1081. Disponible en: https://www.ncbi.nlm.nih.gov/pmc/articles/PMC7144611/

2. Katulanda, P., Dissanayake, H.A., Ranathunga, I. et al. Prevention and management of COVID-19 among patients with diabetes: an appraisal of the literature. Diabetologia. 2020; 63: 1440-1452. Disponible en:https://link.springer.com/article/10.1007/s00125-020-05164-x\#citeas

3. Guan WJ, Ni ZY, Hu Y, Liang WH, Ou CQ, He JX, Liu L, Shan H, Lei CL, Hui DSC, Du B, Li LJ, Zeng G, Yuen KY, Chen RC, Tang CL, Wang T, Chen PY, Xiang J, Li SY, Wang JL, Liang ZJ, Peng YX, Wei L, Liu Y, Hu YH, Peng P, Wang JM, Liu JY, Chen Z, Li G, Zheng ZJ, Qiu SQ, Luo J, Ye CJ, Zhu SY, Zhong NS; China Medical Treatment Expert Group for Covid-19. Clinical Characteristics of Coronavirus Disease 2019 in China. N Engl J Med. 2020 Apr 30;382(18):1708-1720. doi: 10.1056/NEJMoa2002032. Epub 2020 Feb 28. PMID: 32109013; PMCID: PMC7092819.

4. Hernández A. Por qué los diabéticos son grupo de riesgo frente a la infección COVID-19. Revista cubana de Medicina. 2019; 58(2): 1. Disponible en: https://articulos.sld.cu/diabetes/

5. Lima-Martínez MM, Carrera Boada C, Madera-Silva MD, Marín W, Contreras M. COVID-19 and diabetes: A bidirectional relationship. Clin Investig Arterioscler. 2021 May-Jun;33(3):151-157. English, Spanish. doi: 10.1016/j.arteri.2020.10.001. Epub 2020 Oct 28. PMID: 33303218; PMCID: PMC7598432.

6. Moraga C Javier, Cartes-Velásquez Ricardo. PAUTAS DE CHEQUEO, PARTE II: QUOROM Y PRISMA. Rev Chil Cir. 2015 ; 67(3): 325-330. Disponible en: http://www.scielo.cl/scielo.php?script=sci_arttext\&pid=S071840262015000300015\&lng=es. http://dx.doi.org/10.4067/S0718-40262015000300015.

7. Awadhesh K, Ritesh G, Amerta G, Anoop M. Diabetes in COVID-19: Prevalence, pathophysiology, prognosis and practical considerations. Journal Diabetes Metab Syndr. 2020; 14 (4): 303-310. Disponible en: https://www.ncbi.nlm.nih.gov/pmc/articles/PMC7195120/

8. Kosinski C, Zanchi A, Wojtusciszyn A. Diabète et infection à COVID-19. Rev Med Suisse. 2020; 6(16): 939-943. Disponible en: https://pubmed.ncbi.nlm.nih.gov/32374541/

9. Rimesh P, Sanjay K. COVID-19 and diabetes mellitus: An unholy interaction of two pandemics. Diabetes Metab Syndr. 2020;14 (4): 513-517. Disponible en: https://www.sciencedirect.com/science/article/abs/pii/S1871402120301144

10. Czupryniak L. Diabetes mellitus e infección por SARS-CoV-2: riesgo, curso e indicaciones de actuación. Revista Médica Empendium. 2020;(5): 41-45. Disponible en: https://empendium.com/manualmibe/covid19/238194,diabetes-mellitus-einfeccion-por-sars-cov-2-riesgo-curso-e-indicaciones-de-actuacion

11. Chen Y, Yang D, Cheng B, Chen J, Peng A, Yang C, Liu C, Xiong M, Deng A, Zhang Y, Zheng L, Huang K. Clinical Characteristics and Outcomes of Patients With Diabetes and COVID-19 in Association With Glucose-Lowering Medication. Diabetes Care. 2020; 43(7): 1399-1407. Disponible en: https://pubmed.ncbi.nlm.nih.gov/32409498/ 
12. Fernández García L, Puentes Gutiérrez A, García Bascones M. Relación entre obesidad, diabetes e ingreso en UCl en pacientes COVID-19. Revista Medicina Clínica. 2020; 6(9): 314-315. Disponible en: https://www.elsevier.es/es-revistamedicina-clinica-2-avance-resumenrelacion-entre-obesidad-diabetes-e-S0025775320303985?newsletter=true\&coronavirus

13. . Guo W, Li M, Dong Y, al e. Diabetes is a risk factor for the progression and prognosis of COVID-19. Diabetes Metab Res Rev. 2020; 36 (7): 1-8. Disponible en: https://onlinelibrary.wiley.com/doi/full/10.1002/dmrr.3319

14. Hartmann Boyce J, Morris E, Goyder C, Kinton J, Perring J, Nunan D, et al. Diabetes and risks from COVID-19. CEBM. 2020: 1-10. Disponible en: https://www.cebm.net/covid-19/diabetes-and-risks-from-covid-19/

15. Hussain A, Bhowmik B, do Vale Moreira NC. COVID-19 and diabetes: Knowledge in progress. Diabetes Res Clin Pract. 2020 Apr;162:108142. doi: 10.1016/j.diabres.2020.108142. Epub 2020 Apr 9. PMID: 32278764; PMCID: PMC7144611.

16. Kumar, A., Arora, A., Sharma, P., Anikhindi, S., Bansal, N., Singla, V, Srivastava, A. Is diabetes mellitus associated with mortality and severity of COVID-19? A meta-analysis. Diabetes \& Metabolic Syndrome: Clinical Research \& Reviews. 2020; 14(4): 535-545.

17. Kumar A, Gupta R, Ghosh A, Misra A. Diabetes in COVID-19: Prevalence, pathophysiology, prognosis and practical considerations. Diabetes Metab Syndr. 2020; 14 (4): 303-310. Disponible en: https://pubmed.ncbi.nlm.nih.gov/32298981/

18. $\mathrm{Ma} \mathrm{R}$, Holt R. COVID-19 and diabetes. Diabetic Medicine. 2020; 37(5): 723-725. Disponible en: https://onlinelibrary.wiley.com/doi/full/10.1111/dme.14300

19. Zachary T. Diabetes and COVID-19. Journal of Diabetes. 2020;(12): $347-349 . \quad$ Disponible en: https://onlinelibrary.wiley.com/doi/epdf/10.1111/1753-0407.13027

20. Gómez A, Del Castillo S, Chiu E, Salsavilca E. Crisis hiperglicémica aguda como debut de diabetes mellitus y COVID-19: ¿Una nueva causa de diabetes? Pre Print. 2020;(1): 1-6. Disponible en: https://doi.org/10.1590/SciELOPreprints.1135

21. Paquot N, Radermecker R. COVID-19 and diabetes. Rev Med Liege. 2020; 75(1): 138-145. Disponible en: https://pubmed.ncbi.nlm.nih.gov/33211437/

22. Feldman E, Savelieff M, Hayek S, Pennathur S, Kretzler M, Pop-Busui R. COVID-19 and Diabetes: A Collision and Collusion of Two Diseases. Diabetes. Diabetes. 2020; 69(12): 2549-2565.

23. Lima M, Carrera C, Madera M, Marín W, Contreras M. COVID-19 and diabetes: A bidirectional relationship. Clin Investig Arterioscler. 2020; 9168(20): 30105- 30104. Disponible en:https://pubmed.ncbi.nlm.nih.gov/33303218/21.

24. Palaiodimos L, Chamorro-Pareja N, Karamanis D, Li W, Zavras PD, Chang KM, Mathias P, Kokkinidis DG. Diabetes is associated with increased risk for in-hospital mortality in patients with COVID-19: a systematic review and meta-analysis comprising 18,506 patients. Hormones (Athens). 2021 Jun;20(2):305-314. doi: 10.1007/s42000-020-00246-2. Epub 2020 Oct 29. PMID: 33123973; PMCID: PMC7595056.

25. Medina C, Hernández E. Diabetes Mellitus in the context of COVID-19. Revdosdic. 2020; 3(3): 1-25. Disponible en: http://www.revdosdic.sld.cu/index.php/revdosdic/article/view/81

26. Ranganath M, Sriram G. COVID-19 pandemic, coronaviruses, and diabetes mellitus. 2020; 318: 736-739. Disponible en: https://journals.physiology.org/doi/full/10.1152/ajpendo.00124.202039.

27. Jeong K, Yoon K, Lee M. Diabetes and COVID-19: Global and regional perspectives. Diabetes Res Clin Pract. 2020; 166: 108303. Disponible en: https://pubmed.ncbi.nlm.nih.gov/32623038/

28. Rimesh P, Bhansali A. COVID-19, diabetes mellitus and ACE2: The conundrum. Diabetes research and clinical practice. 2020; 162(108132): 1-3. Disponible en:https://www.diabetesresearchclinicalpractice.com/article/S01688227(20)30382X/fulltext

29. Salazar J. Manejo de pacientes con diabetes en el contexto de la Pandemia COVID-19. Revista de la Pontificia Universidad Católica del Ecuador. 2020: 1-17. Disponible en: https://puceapex.puce.edu.ec/web/covid19-medidaspreventivas/wp-content/uploads/sites/6/2020/06/manejo-de-pacientes-con-diabetes.pdf

30. $\mathrm{Ng} \mathrm{K}$, Rickard J. The Effect of COVID-19 on Patients With Diabetes. US Pharmacist. 2020; 45(11): 9-12. Disponible en: https://www.uspharmacist.com/article/the-effect-of-covid19-on-patients-with-diabetes

31. Chakraborty C, Sharma A, Bhattacharya M, Sharma G, Agoramoorthy G, Lee S. Diabetes and COVID-19: a major challenge in pandemic period? Eur Rev Med Pharmacol Sci. 2020; 24(21): 11409-11420. Disponible en: https://pubmed.ncbi.nlm.nih.gov/33215463/

32. Ugwueze C, Chukwuma B, Nnolim B, Agim E, Anikpo N, Onyekachi K. COVID-19 and Diabetes Mellitus: The Link and Clinical Implications. Dubai diabetes and endocrinology Journal. 2020;(26): 69-75. Disponible en: https://www.karger.com/Article/PDF/511354

33. Henoy A, Ismaily M, Bajaj M. Diabetes and COVID-19 on Patients With Diabetes. US Pharmacist. 2020; 45(11): 9-12.: a global health challenge. BMJ Open diabetes research \& Care. 2020; 8(1450): 1-2. Disponible en: https://drc.bmj.com/content/bmjdrc/8/1/e001450.full.pdf

34. Desai R, Singh S, Parekh T, Sachdeva S, Sachdeva R, Kumar G. COVID-19 and diabetes mellitus: A need for prudence in elderly patients from a pooled analysis. Diabetes Metab Syndr. 2020; 14(4): 683-685. Disponible en: https://pubmed.ncbi.nlm.nih.gov/32438333/

35. Lim S, Hyun J, Sang H, Nauck M. COVID-19 and diabetes mellitus: from pathophysiology to clinical management. Nature endocrinology. 2020;(17): 11-30. Disponible en: https://www.nature.com/articles/s41574-020-00435-4

36. Bellido V, Pérez A. Consecuencias de la COVID-19 sobre las personas con diabetes. Revista de Endocrinología, Diabetes y Nutrición. 2020; 67(6): 355-356. Disponible en: https://www.elsevier.es/es-revista-endocrinologia-diabetes-nutricion-13articulo-consecuencias-covid-19-sobre-personas-con-S253001642030104X

37. Roncón L, Zuin M, Rigatelli G, Zuliani G. Diabetic patients with COVID-19 infection are at higher risk of ICU admission and poor short-term outcome. Journal of Clinical Virology. 2020; 127: 1-3.

38. Paz J. Manejo de la diabetes mellitus en tiempos de COVID-19. Acta Médica Peruana. 2020; 37(2): 176-182. Disponible en: http://www.scielo.org.pe/pdf/amp/v37n2/1728-5917-amp-37-02-176.pdf 
39. Torres M, Caracas N, Peña B, Juárez J, Medina A, Martínez M. Infección por coronavirus en pacientes con diabetes. Cardiovascular and Metabolic Science. 2020; 31(3): 235-244. Disponible en: https://www.medigraphic.com/pdfs/cardiovascuar/cms-2020/cmss203n.pdf

40. Muniangi H, Akalestou E, Salem V, Misra S, Oliver N, Rutter G. COVID-19 and Diabetes: A Complex Bidirectional Relationship. Front Endocrinol. 2020; 8(11): 1-10. Disponible en: https://pubmed.ncbi.nlm.nih.gov/33133024/

41. Fildari T, Morano S. COVID-19: is there a link between the course of infection and pharmacological agents in diabetes? J Endocrinol Invest. 2020; 43(8): 1053-1060. Disponible en: https://pubmed.ncbi.nlm.nih.gov/32495299/

42. Erener S. Diabetes, infection risk and COVID-19. Mol Metab. 2020; 39:101044. Disponible en: https://pubmed.ncbi.nlm.nih.gov/32585364/

43. Bouhanick B, Cracowski J, Faillie J. Diabetes and COVID-19. Therapie Journal. 2020; 75(4): 327-333. Disponible en: https://pubmed.ncbi.nlm.nih.gov/32425249/

44. Peric S, Stulnig T. Diabetes and COVID-19: Disease-Management-People. Wien Klin Wochenschr Journal. 2020; 132: 356361. Disponible en: https://pubmed.ncbi.nlm.nih.gov/32435867/

45. Abdi A, Jalilian M, Ahmadi P, Vlaisavljevic Z. Diabetes and COVID-19: A systematic review on the current evidences. Diabetes Res Clin Pract. 2020;166: 108347. Disponible en: https://pubmed.ncbi.nlm.nih.gov/32711003/

46. Villabona C. COVID-19 and diabetes. Diabetes Research. 2020;(162): 1-2. Disponible en: https://www.diabetesresearchclinicalpractice.com/action/showPdf?pii=S0168-8227\%2820\%2930388-0 